\title{
Mitochondrial protein import: mechanisms, components and energetics
}

\author{
Elisabeth Schwarz, Walter Neupert * \\ Institut für Physiologische Chemie, Universität München, Goethestr. 33, D-80336 München, Germany
}

Received 11 April 1994

\begin{abstract}
The transport of nuclear-encoded proteins from the cytosol into mitochondria is mediated by targeting (signal) sequences present on precursor forms. Most precursors of the mitochondrial matrix possess amino-terminal signals which characteristically contain hydroxylated and basic amino acids and lack acidic residues. With a minority of precursor proteins, internal sequence motifs can direct proteins to the mitochondria (Pfanner, N., Hoeben, P., Tropschug, M. and Neupert, W. (1987) J. Biol. Chem. 262, 14851-14854). The presence of a mitochondrial targeting sequence alone, however, is not sufficient for specific targeting to the organelle and further to the various subcompartments. There is the need for components which recognise the targeting sequences and others which keep the precursor protein in a translocation-competent form. Beyond the recognition step, components are required which mediate translocation across the mitochondrial membranes. Mitochondria possess two translocation machineries, one in the outer membrane and one in the inner membrane. The matrix space harbors a number of factors which participate in the import of proteins, in their unfolding and folding. Energy is required at several steps of these processes.
\end{abstract}

Key words: Protein import; Mitochondrion; Cytochrome biogenesis; Chaperone; Import receptor; Protein folding

\section{The receptor complex of the outer membrane}

Early investigations mainly concentrated on the characterisation of the proteins in the outer membrane involved in precursor import. Mild protease treatment of Neurospora crassa mitochondria impaired protein import to a large extent. Thus, it was concluded that protease-sensitive components in the outer membrane are required for this process [2]. Subsequently, these receptors were identified as proteins of 72 and $19 \mathrm{kDa}$ (MOM72 and MOM19) [3,4]. By inhibiting the protein import with IgG against the respective receptors it was demonstrated that the ADP/ATP carrier primarily uses MOM72 whereas the majority of precursor proteins are recognised by MOM19. Still the two receptors appear to overlap in their specificity. MOM72 consists of two domains. The $\mathrm{N}$-terminal $12 \mathrm{kDa}$ domain provides the membrane anchor, whereas the $\mathrm{C}$-terminal 60 $\mathrm{kDa}$ domain is exposed to the cytosol. The recombi-

\footnotetext{
${ }^{*}$ Corresponding author. Fax: +49895996270.
}

nant cytosolic domain is able to bind the precursors of the ADP/ATP carrier, the phosphate carrier and cytochrome $c_{1}$ and thereby competes for import of these precursors [5]. Cloning and sequence analysis of the yeast homologue of MOM72, MAS70, showed a high conservation between the two proteins. The overlapping specifity of the two receptors, MOM19 and MOM72, could be demonstrated by gene disruption of the yeast homologue, MAS70, which did not result in a gross respiratory deficiency, probably because it can be partially replaced by MAS20, the yeast homologue of MOM19 [6,7]. Gene disruption of MAS20 caused loss of respiration, which could be rescued by overexpression of MAS70, again demonstrating the overlapping function [7]. Inactivation of the $N$. crassa MOM19 gene has recently been achieved by a novel technique designed to introduce mutations into $N$. crassa genes [8]. The characterisation of these mutant $N$. crassa cells demonstrated the important role of MOM19 in precursor import: cells in which the MOM19 gene was inactivated stopped to grow, the mitochondria lost their protein synthesis activity as well as their respiratory complexes [9]. 
Lysis of $N$. crassa mitochondria with mild detergents and subsequent immunoprecipitation with antiMOM19 antibodies revealed that MOM19 is present in a complex with MOM72 and a number of other proteins of 7, 8, 22 and $38 \mathrm{kDa}$ (MOM7, MOM8, MOM22 and MOM38) [10]. MOM38 turned out to be considerably more proteinase-resistant than the two receptors, MOM19 and MOM72. The primary sequence suggests at least one potential membrane spanning region and a putative ATP binding domain [11]. The yeast homologue ISP42 (Import Site Protein of $42 \mathrm{kDa}$ ) is essential for cell viability [12]. Antibodies against $N$. crassa MOM38 can inhibit the assembly of $N$. crassa MOM19 into the outer membrane of isolated yeast mitochondria reflecting the high evolutionary conservation of the mitochondrial import system [13]. In a genetic approach ISP6 was characterised as a multi-copy suppressor for the mutant ISP42 allele [14]. ISP6 consists of 61 amino acid residues and probably spans the outer membrane with the $\mathrm{N}$-terminus oriented towards the the cytosol. So far the precise role of ISP6 and its relation to MOM7 anbd MOM8 in the translocation process remains to be elucidated.

A hypothesis on the mechanisms of protein transfer across the outer membrane can be formulated on the basis of biochemical studies: Precursor proteins by the virtue of their $\mathrm{N}$-terminal or internal targeting sequences bind to MOM19 and/or MOM72 and are subsequently transferred to translocation contact-sites where the two membranes are in close contact. Import studies with the ADP / ATP carrier elucidated the role of MOM22, a protein which spans the outer membrane once [15]. MOM22 appears to promote the initial entrance of the receptor-bound precursors into a General Insertion Pore (GIP) which consists of MOM38 and probably MOM7, MOM8 in $N$. crassa, or ISP42, ISP6 and probably other components in $S$. cerevisiae. The GIP-complex can be co-immunoprecipitated with antibodies against MOM19 [10]. Evidence for the existence of a general insertion pore has been obtained from the observation that this site can be saturated by chemical amounts of porin precursors [16] and that the ADP/ATP carrier in the absence of a membrane potential can accumulate at the GIP-site in a proteinase-protected location [17].

The question of how preciseley proteins are translocated across the outer membrane still requires intensive investigation. An initial study with outer membrane vesicles demonstrated that they possess a translocation activity on their own. However, translocation into outer membrane vesicles is restricted to those proteins which are integrated into the outer membrane and to a limited set of those proteins which are translocated into the intermembrane space [18]. It should be emphasised that this translocation activity does not require components of the inner membrane, and is restricted to proteins which can be imported into mitochondria in the absence of a membrane potential.

Thus, the elucidation of the mechanism of protein translocation across the outer membrane through the receptor-GIP-complex probably will yield a quite complex picture. The question arises as to the evolutionary origin of such an intricate machinery. It remains to be determined whether the outer membrane translocation machinery has evolved from the eukaryotic host or is derived from the original protosymbiontic cell. No bacterial homologues are known so far for the proteins of the complex characterised. Interesting in this context is the observation that a mitochondrial presequence can be imported into Paracoccus denitrificans cells [19].

\section{The import machinery of the inner membrane}

The identification of components involved in protein translocation across the inner membrane has begun only recently. MPI1 has been identified as the first protein of the inner membrane involved in precursor import [20-22]. MPI1 or ISP45 was later renamed MIM44 to make clear that it is an inner membrane protein. Its substantial role in precursor import was confirmed by cross-linking with a translocation intermediate [23]. MIM44 is associated with the inner face of the inner membrane and cooperates with mitochondrial Hsp70 in promoting import of precursor proteins (Schneider, Berthold, Brunner and Neupert, submitted). Genetic approaches revealed that two more proteins, MAS6 (MIM23) and MIM17, are involved in the translocation across the inner membrane [24,25]. The role of MAS6 in precursor import could be confirmed by cross-linking MAS6 to an arrested precursor protein [26].

The import machineries of the outer and inner membrane act in a coordinated manner to translocate precursor proteins across the two mitochondrial membranes. However, according to recent investigations, they can also function independently: (i) isolated outer membrane vesicles possess translocation activity for a subset of proteins [18]; (ii) fusion proteins consisting of a matrix-targeting sequence connected to the enzyme CCHL can be imported into the intermembrane space in the absence of a membrane potential and in a second step, after restoration of the membrane potential, the fusion protein can be imported into the matrix [27].

\section{Role of mitochondrial heat-shock proteins in precur- sor translocation, protein unfolding and folding}

An essential role in mitochondrial biogenesis has recently been demonstrated for the matrix-localised 
heat-shock proteins. An Hsp70 homologue present in yeast mitochondria is encoded by the essential SSC1 gene [28]. The analysis of cells with a temperature-sensitive allele of $\mathrm{Hsp} 70$ revealed that the protein plays an essential role in precursor import [29]. Very recently a mitochondrial homologue of the bacterial GrpE, YGE1, has been identified by a genetic screen for staurosporine-resistant clones in $S$. cerevisiae [30]. Depletion of YGE1 resulted in the loss of mitochondrial DNA and finally cell viability. It is very likely that YGE1 together with Hsp70 mediates precursor import into the matrix. The requirement of matrix ATP for the import reaction reflects, at least partly, the essential action of $\mathrm{Hsp} 70$ in this process. Depletion of matrix ATP inhibits import across the inner membrane at an early stage [31-34]. It could be demonstrated that the requirement of ATP reflects the extent of folded structure which has to be resolved in order for the import reaction to take place as translocation across membranes requires the protein to assume an extended conformation [35,36]. Recently, a mitochondrial homologue of bacterial DnaJ, MDJ1 (Mitochondrial DnaJ), has been characterised in $S$. cerevisiae. Interestingly, MDJ1 does not play an essential role in protein import. Mitochondria from the MDJ1 deletion strain do import precursor proteins, however, the folding to the native conformation is affected, in particular at higher temperatures [37]. It remains to be determined whether MDJ1 cooperates with Hsp70 in the folding process or whether it can interact by itself with the unfolded polypeptide chains.

The final step of protein folding is mediated by Hsp60 in an ATP-dependent manner [38]. Disruption of the Hsp60 encoding MIF4 gene is lethal indicating its essential function in mitochondrial biogenesis. Mitochondria harboring a temperature-sensitive Hsp60 allele accumulated unfolded proteins in the matrix [39]. Thus, the two mitochondrial heat-shock proteins, Hsp70 and Hsp60, appear to act in a sequential process: Hsp70 mediates the translocation of precursor proteins across the membranes, Hsp60 promotes folding to the native conformation and assembly into oligomeric complexes [40]. As expected, mitochondrial Hsp60 interacts with its cognate Hsp10 which was recently identified [41,42]. Disruption of the gene is lethal stressing its important function in protein folding reactions which has been demonstrated with the purified components [43].

\section{Bioenergetics of mitochondrial protein import and sorting}

As described above nuclear-encoded proteins have to face a series of interactions with components of the mitochondrial protein import and folding machinery until they become finally functional in mitochondria. We briefly rehearse the sequence of events from the synthesis in the cytosol to the finally functional protein with emphasis on the bioenergetic aspects of the processes.

(1) After their synthesis in the cytosol, proteins have to be maintained in a translocation-competent form. This translocation-competent conformation is mediated at least partly by cytosolic heat-shock proteins of the Hsp70 class [44]. The release from the Hsp70s depends on the presence of hydrolysable ATP and probably other cytosolic factors. Extramitochondrial ATP has been shown to be required for the import of a number of precursor proteins. This very likely reflects the extent of their association with Hsp70 molecules [45].

(2) After interaction with the mitochondrial surface receptors protein translocation into or across the mitochondrial membranes occurs. Presently, it is still unclear whether the translocation process across the outer membrane itself comprises an ATP-consuming reaction. Insertion into and translocation across the outer membrane does not require a membrane potential across the inner membrane as it is possible to accumulate the ADP/ATP carrier at the GIP-site [17]. Furthermore, certain proteins can be translocated across the outer membrane in the absence of a membrane potential [27]. The insertion of the presequence into the inner membrane, however, strictly depends on a membrane potential $\Delta \psi$ [46]. Completion of translocation is independent of an energised inner membrane.

(3) Translocation of the presequence of matrixtargeted proteins strictly depends on the action of Hsp70 in the mitochondrial matrix and the presence of ATP. When matrix ATP is depleted the presequence can slip back out of the translocation channel [34]. Thus, the membrane potential allows a reversible insertion of the presequence into the inner membrane. If the translocation channel facilitates reversible movement of the precursor polypeptide and if Hsp70s bind to segments of the precursor both on the cytosolic and mitochondrial matrix side, the question arises as to why a vectorial transport into the matrix occurs. MIM44 in the matrix may constitute one of the additional import components required by mt-Hsp70s to mediate a directional import reaction. It is an open question how many cycles of binding to $\mathrm{Hsp} 70$ a given protein has to undergo in order to be completely imported into the matrix. Another problem is whether folding occurs concomitant with the import reaction or whether the final folding process mediated by Hsp60 starts only after complete translocation into the matrix.

(4) Whereas protein import into the matrix appears to occur along a general pathway, several import routes are taken by the proteins destined for the intermembrane space. Apocytochrome $c$ is able to penetrate the 
outer membrane without assistance of the receptorGIP-complex and appears to be trapped in the intermembrane space after the covalent attachment of heme [47]. This process is catalysed by the enzyme cytochrome $c$ heme lyase (CCHL), located on the surface of the inner membrane facing the intermembrane space. The import of CCHL itself is an interesting problem. Transport of CCHL occurs directly across the outer membrane and requires the receptor-GIP complex but is not dependent on a membrane potential. At the same time, extramitochondrial ATP does not appear to be necessary, implying that the CCHL precursor does not bind to cytosolic heat-shock proteins [48]. Possibly, the driving force for import is the folding/assembly reaction which occurs in the intermembrane space. It remains to be determined whether this folding requires chaperones and/or other components docking CCHL to the membrane.

Cytochrome $b_{2}$, a soluble protein of the intermembrane space, and cytochrome $c_{1}$, which is anchored to the inner membrane by its $\mathrm{C}$-terminus and faces the intermembrane space, possess a bipartite presequence. The first part of the presequence represents a typical mitochondrial targeting sequence. The second part ('sorting sequence') directs the protein to the intermembrane space and resembles bacterial leader sequences. For the intramitochondrial sorting of these proteins two models are being discussed: (i) according to the 'conservative sorting' mechanism the protein is first imported into the matrix and then, in a second step, retranslocated across the inner membrane [49]; (ii) alternatively, the protein would become arrested in the inner membrane by the sorting sequence ('stoptransfer') and directly be released to the intermembrane space [50].

Conservative sorting of another protein anchored to the inner membrane and facing the intermembrane space, the Rieske-iron-sulfur protein $(\mathrm{FeS})$, was confirmed by the identification of an intermediate form in the matrix, where it occurs soluble, unassembled and associated with mt-Hsp70 (Fölsch, Stuart and Neupert, unpublished data). Consequently, the import and assembly of $\mathrm{FeS}$ requires matrix ATP.

More difficult to understand is the matrix ATP requirement for the sorting of cytochrome $c_{1}$ and $b_{2}$. Cytochrome $c_{1}$ is directed to the intermembrane space even after matrix ATP has been depleted [51]. This result can be interpreted in two ways: (i) the protein is sorted by stop-transfer without passage through the matrix; (ii) the import of cytochrome $c_{1}$ through the outer and inner membranes is driven by another energy source, e.g., by taking place concomitantly with the export across the inner membrane. In contrast to cytochrome $c_{1}$, import of full-length cytochrome $b_{2}$ requires ATP. Short fusion proteins of up to 167 amino acids of cytochrome $b_{2}$ connected to DHFR (dihydro- folate-reductase) were sorted into the intermembrane space even after matrix ATP depletion, whereas fusion proteins with longer cytochrome $b_{2}$ sequences depended on matrix ATP for import and translocation into the intermembrane space. Since unfolding of these constructs by urea prior to import abolished ATP dependence as did the deletion of the tightly folded heme domain, it was concluded that the ATP requirement resulted from the folded structure still outside the mitochondria [36]. Mt-Hsp70 could facilitate unfolding by binding to the incoming polypeptide chain and shifting the equilibrium of the folded and unfolded state caused by spontaneous partial unfolding. The difference in ATP requirement for matrix- and intermembrane space-targeted proteins became obvious by the analysis of two short cytochrome $b_{2}$ DHFR fusion constructs, which only differ in the intramitochondrial sorting sequence, i.e., a deletion of 19 amino acids. The deletion causes missorting into the matrix space. Thus, import of the deletion construct into the matrix depended on matrix ATP in contrast to the construct with the intact sorting sequence which was sorted to the intermembrane space also in the absence of ATP. Apparently, sorting to the intermembrane space can drive import and render it ATP-independent, unless tightly folded domains need to be resolved.

\section{Acknowledgements}

This work was supported by grants from the Deutsche Forschungsgemeinschaft Ne 438/2-1, Sonderforschungsbereich 184, Bonn, Fonds der Chemischen Industrie, Frankfurt, and the EC Human Capital and Mobility Programme, Brussels.

\section{References}

[1] Pfanner, N., Hoeben, P., Tropschug, M. and Neupert, W. (1987) J. Biol. Chem. 262, 14851-14854.

[2] Zwizinskì, C., Schleyer, M. and Neupert, W. (1984) J. Biol. Chem. 259, 7850-7856.

[3] Söllner, T., Griffiths, G., Pfaller, R., Pfanner, N. and Neupert W. (1989) Cell 59, 1061-1071.

[4] Söllner, T., Pfaller, R., Griffiths, G., Pfanner, N. and Neupert, W. (1990) Cell 62, 107-115.

[5] Schlossmann, J., Dietmeier, K., Pfanner, N. and Neupert, W. (1994) J. Biol. Chem. in press.

[6] Steger, H.F., Söllner, T., Kiebler, M., Dietmeier, K.A., Pfaller, R., Trülzsch, K.S., Tropschug, M., Neupert, W. and Pfanner, N. (1990) J. Cell Biol. 111, 2353-2363.

[7] Ramage, L., Junne, T., Hahne, K., Lithgow, T. and Schatz, G. (1993) EMBO J. 12, 4115-4123.

[8] Harkness, T.A.A., Metzenberg, R.L., Schneider, H., Lill, R., Neupert, W. and Nargang, F.E. (1994) Genetics 136, 107-118.

[9] Harkness, T.A.A., Nargang, F.E., van der Klei, I., Neupert, W. and Lill, R. (1994) J. Cell Biol. 124, 637-648. 
[10] Moczko, M., Dietmeier, K., Söllner, T., Segui-Real, B., Steger, H.F., Neupert, W. and Pfanner, N. (1992) FEBS Lett. 310, 265-268.

[11] Kiebler, M., Pfaller, R., Söllner, T., Griffiths, G., Horstmann, H., Pfanner, N. and Neupert, W. (1990) Nature 348, 610-616.

[12] Baker, K.P., Schaniel, A., Vestweber, D. and Schatz, G. (1990) Nature 348, 605-609.

[13] Schneider, H., Söllner, T., Dietmeier, K., Eckerskorn, C., Lottspeich, F., Trülzsch, B., Neupert, W. and Pfanner, N. (1991) Science 254, 1659-1662.

[14] Kassenbrock, C.K., Cao, W. and Douglas, M.G. (1993) EMBO J. 12, 3023-3034.

[15] Kiebler, M., Keil, P., Schneider, H., Van der Klei, I., Pfanner, N. and Neupert, W. (1993) Cell 74, 483-492.

[16] Pfaller, R., Steger, H.F., Rassow, J., Pfanner, N. and Neupert, W. (1988) J. Cell Biol. 107, 2483-2490.

[17] Söllner, T., Rassow, J., Wiedmann, M., Schlossmannn, J., Keil, P., Neupert, W. and Pfanner, N. (1992) Nature 355, 84-87.

[18] Mayer, A., Lill, R. and Neupert, W. (1993) J. Cell Biol. 121, 1233-1243.

[19] Roise, D. and Maduke, M. (1994) FEBS Lett. 337, 9-13.

[20] Maarse, A.C., Blom, J., Grivell, L.A. and Meijer, M. (1992) EMBO J. 11, 3619-3628.

[21] Scherer, P.E., Manning-Krieg, U.C., Jenö, P., Schatz, G. and Horst, M. (1992) Proc. Natl. Acad. Sci. USA 89, 11930-11934.

[22] Horst, M., Jenö,P., Kronidou, N.G., Bolliger, L., Opplinger, W., Scherer, P., Manning-Krieg, U., Jascur, T. and Schatz, G. (1993) EMBO J. 12, 3035-3941.

[23] Blom, J., Kübrich, M., Rassow, J., Voos, W., Dekker, P.J.T., Maarse, A.C., Meijer, M. and Pfanner, N. (1993) Mol. Cell. Biol. $13,7364-7371$.

[24] Emtage, J.L.T. and Jensen, R.E. (1993) J. Cell Biol. 122, $1003-$ 1012.

[25] Dekker, P.J.T. Keil, P., Rassow, J., Maarse, A.C., Pfanner, N. and Meijer, M. (1993) FEBS Lett. 330, 66-70.

[26] Ryan, K.R. and Jensen, R.E. (1993) J. Biol. Chem. 268, 2374323746.

[27] Segui-Real, B., Kispal, G., Lill, R. and Neupert, W. (1993) EMBO J. 12, 2211-2218.

[28] Craig, E.A., Kramer, J., Shilling, J., Werner-Washburne, M., Holmes, S., Kosic-Smithers, J. and Nicolet, C.M. (1989) Mol. Cell. Biol. 9, 3000-3008.

[29] Kang, P.-J., Ostermann, J., Shilling, J., Neupert, W., Craig, E.A. and Pfanner, N. (1990) Nature 348, 137-143.
[30] Ikeda, E., Yoshida, S., Mitsuzawa, H., Uno, I. and Toh-e, A. (1994) FEBS Lett. 339, 265-268.

[31] Hwang, S.T., Wachter, C. and Schatz, G. (1991) J. Biol. Chem. 266, 21083-21089.

[32] Rassow, J. and Pfanner, N. (1991) FEBS Lett. 293, 85-88.

[33] Jascur, T., Goldenberg, D.P., Vestweber, D. and Schatz, G. (1992) J. Biol. Chem. 267, 13636-13641.

[34] Cyr, D.M., Stuart, R.A. and Neupert, W. (1993) J. Biol. Chem. 268, 23751-23754.

[35] Rassow, J., Hartl, F.-U., Guiard, B., Pfanner, N. and Neupert, W. (1990) FEBS Lett. 275, 190-194.

[36] Stuart, R.A., Gruhler, A., Van der Klei, I., Guiard, B., Koll, H. and Neupert, W. (1994) Eur. J. Biochem. 220, 9-18.

[37] Rowley, N., Prip-Buus, C., Westermann, B., Brown, C., Schwarz, E., Barrell, B. and Neupert, W. (1994) Cell 77, 249-259.

[38] Ostermann, J., Horwich, A.L., Neupert, W. and Hartl, F.-U. (1989) Nature 341, 125-130.

[39] Cheng, M.Y., Hartl, F.-U., Martin, J., Pollock, R.A., Kalousek, F., Neupert, W., Hallberg, E.M., Hallberg, R.L. and Horwich, A.L. (1989) Nature $337,620-625$.

[40] Manning-Krieg, U.C., Scherer, P.E. and Schatz, G. (1991) EMBO J. 10, 3273-3280.

[41] Rospert, S., Junne, T., Glick, B.S. and Schatz, G. (1993) FEBS Lett. 335, 358-360.

[42] Höhfeld, J. and Hartl, F.-U. (1994) J. Cell Biol., submitted.

[43] Rospert, S., Glick, B.S., Jenö, P., Schatz, G., Todd, M.J., Lorimer, G.H. and Viitanen, P.V. (1993) Proc. Natl. Acad. Sci. USA 90, 10967-10971.

[44] Deshaies, R.J., Koch, B.D., Werner-Washburne, M., Craig, E.A. and Schekman, R. (1988) Nature 332, 800-805.

[45] Pfanner, N., Rassow, J., Guiard, B., Söllner, T., Hartl, F.-U. and Neupert, W. (1990) J. Biol. Chem. 265, 16324-16329.

[46] Zwizinski, C., Schleyer, M. and Neupert, W. (1984) J. Biol. Chem. 258, 4071-4074.

[47] Nicholson, D.W., Hergersberg, C. and Neupert, W. (1988) J. Biol. Chem. 263, 19034-19042.

[48] Lill, R., Stuart, R.A., Drygas, M.E., Nargang, F.E. and Neupert, W. (1992) EMBO J. 11, 449-456.

[49] Hartl, F.-U., Ostermann, J., Guiard, B. and Neupert, W. (1987) Cell 51, 1027-1037.

[50] Glick, B.S., Brandt, A., Cunningham, K., Müller, S., Hallberg, R.L. and Schatz, G. (1992) Cell 69, 809-822.

[51] Wachter, C., Schatz, G. and Glick, B.S. (1992) EMBO J. 11, 4787-4794. 